\title{
Inorganic semiconductor nanomaterials for flexible and stretchable bio-integrated electronics
}

\author{
Dae-Hyeong Kim ${ }^{1}$, Nanshu Lu², Roozbeh Ghaffari ${ }^{3}$ and John A Rogers ${ }^{4}$ \\ Rapid advances in semiconductor nanomaterials, techniques for their assembly, and strategies for incorporation into functional \\ systems now enable sophisticated modes of functionality and corresponding use scenarios in electronics that cannot be \\ addressed with conventional, wafer-based technologies. This short review highlights enabling developments in the synthesis \\ of one- and two-dimensional semiconductor nanomaterials (that is, NWs and nanomembranes), their manipulation and use in \\ various device components together with concepts in mechanics that allow integration onto flexible plastic foils and stretchable \\ rubber sheets. Examples of systems that combine with or are inspired by biology illustrate the current state-of-the-art in this \\ fast-moving field.
}

NPG Asia Materials (2012) 4, e15; doi:10.1038/am.2012.27; published online 20 April 2012

Keywords: bio-integrated electronics; flexible electronics; semiconductor nanomaterials; stretchable electronics; transfer printing

\section{INTRODUCTION}

Research in semiconductor nanomaterials, starting with foundational work on nanocrystals ${ }^{1,2}$ and fullerenes ${ }^{3}$ in the 1980s, represents a continuing, central thrust of activity in nanoscience and nanotechnology. Interest derives from the many attractive attributes in charge transport, light emission, mechanics and thermal diffusion that emerge at nanometer dimensions, due explicitly to size scaling effects. ${ }^{4}$ The most widely explored material geometries include zero-, one- and two-dimensional configurations (that is, zero-dimensional dots; one-dimensional wires and two-dimensional membranes, respectively), formed by diverse techniques in chemical synthesis, ${ }^{5-7}$ in self-assembled growth ${ }^{8-10}$ and in advanced processing of bulk or layered wafers. ${ }^{11-13}$ Although many application areas have been contemplated, some of the most recent and most sophisticated examples are in unusual format electronics, ${ }^{14-16}$ where onedimensional and two-dimensional semiconductor nanomaterials provide uniform, single crystalline pathways for charge transport between lithographically defined contact electrodes. By comparison with analogous, wafer-based technologies, these systems are important in part, because the nanomaterials themselves create engineering options in heterogeneous designs and mechanically flexible/stretchable formats that would be otherwise impossible to achieve. These features follow directly from three critical aspects of mechanics at nanoscale dimensions. First, the bending stiffness of a sheet of material is proportional to the cube of its thickness. ${ }^{17}$ Second, for a given bending radius $(r)$, the induced peak strains decrease linearly with thickness. ${ }^{17}$ These two scaling trends combine to alter, in a simple but dramatic way, the physical nature of nanoscale materials compared with bulk ones. For example, a silicon nanomembrane ( $\mathrm{Si}$ $\mathrm{NM}$ ) with thickness of $10 \mathrm{~nm}$ has a bending stiffness that is 15 orders of magnitude smaller than that of a silicon wafer with thickness $1 \mathrm{~mm}$. The same Si NM can bend to a radius $(r)$ of $0.5 \mu \mathrm{m}$ before reaching the fracture threshold $(\sim 1 \%)$, whereas a wafer can only bend to $r=5 \mathrm{~cm}$. In other words, one-dimensional and twodimensional nanomaterials are extremely flexible and they can be bent with minimal applied force; that is, they are mechanically floppy. A third important feature of the mechanics follows from a linear reduction in the propensity for crack formation (that is, the energyrelease rate for fracture) with thickness. ${ }^{17}$ As a result, by comparison with their bulk counterparts, nanomaterials can more reliably and robustly bond with one another and with other materials; that is, they are sticky in this sense.

These attributes provide routes to high quality flexible/stretchable electronics, in which semiconductor nanomaterials serve as the active components, and plastic sheets or rubber slabs provide compliant substrates. When implemented with optimized mechanics designs, the result is a type of technology that can accommodate exceptionally large deformations with reversible, linear elastic responses to applied force. ${ }^{15}$ Electronics with these properties have wide ranging potential applications, from flexible displays to lightweight, large-area communication systems to thin, conformable structural health monitors. ${ }^{18,19}$ An area of compelling opportunity is in the intimate

\footnotetext{
${ }^{1}$ School of Chemical and Biological Engineering, Institute of Chemical Processes, Seoul National University, Seoul, Korea; ${ }^{2}$ Department of Aerospace Engineering and Engineering Mechanics, University of Texas at Austin, Austin, TX, USA; ${ }^{3}$ MC10 Inc., Cambridge, MA, USA and ${ }^{4}$ Department of Materials Science and Engineering, Beckman Institute for Advanced Science and Technology, and Frederick Seitz Materials Research Laboratory, University of Illinois at Urbana-Champaign, Urbana, IL, USA Correspondence: JA Rogers, Department of Materials Science and Engineering, Beckman Institute for Advanced Science and Technology, and Frederick Seitz Materials Research Laboratory, University of Illinois at Urbana-Champaign, Urbana, IL 61801, USA.

E-mail: jrogers@uiuc.edu
}

Received 2 March 2012; accepted 26 March 2012 
integration of electronics with the soft, curvilinear surfaces of the human body, for advanced diagnostic or therapeutic purposes. ${ }^{20,21}$ In the following, we review recent work on the synthesis of single crystalline one-dimensional and two-dimensional semiconductor nanomaterials suitable for these sorts of applications, and then describe techniques that have shown promise for their assembly into functional systems. Examples of several classes of devices, with an emphasis on those that involve bio-integrated operation, capture the current state of the field and suggest some directions for future work.

\section{SYNTHESIS}

Nanowires (NWs) represent one of the most widely explored class of semiconductor nanomaterials of interest primarily owing to their attractive electronic and optoelectronic properties, but also for the purposes of this review, their mechanical characteristics. ${ }^{22-25}$ Synthesis of NWs from gaseous precursors can be achieved by vapor-liquid-solid processes, first developed by Wagner et al. ${ }^{26}$ and reviewed elsewhere, ${ }^{27,28}$ in which growth results from the gas phase delivery of precursors to the surface of catalytic metal clusters (diameters $\sim 100 \mathrm{~nm}$ or less) at temperatures that exceed the eutectic point. Single crystalline NWs grow epitaxially from the surfaces of these clusters after the reacting species reach their saturated concentration in the metal. Introducing dopants into the reaction vessel can lead to their controlled incorporation into the growing NWs. ${ }^{22,23}$ Switching precursors during growth provides a route to NWs with heterogeneous, core-shell or super lattice structures. $^{22,23}$ These approaches are applicable to a wide range of materials, including group IV, III-V and II-VI semiconductors (Figures 1a and b). ${ }^{29,30}$ NWs of III-V semiconductors with axially or radially modulated dopant concentrations provide an additional flexibility in design for advanced optoelectronic components, including solar cells, light-emitting diodes (LEDs) and laser diodes. ${ }^{22,31-33}$
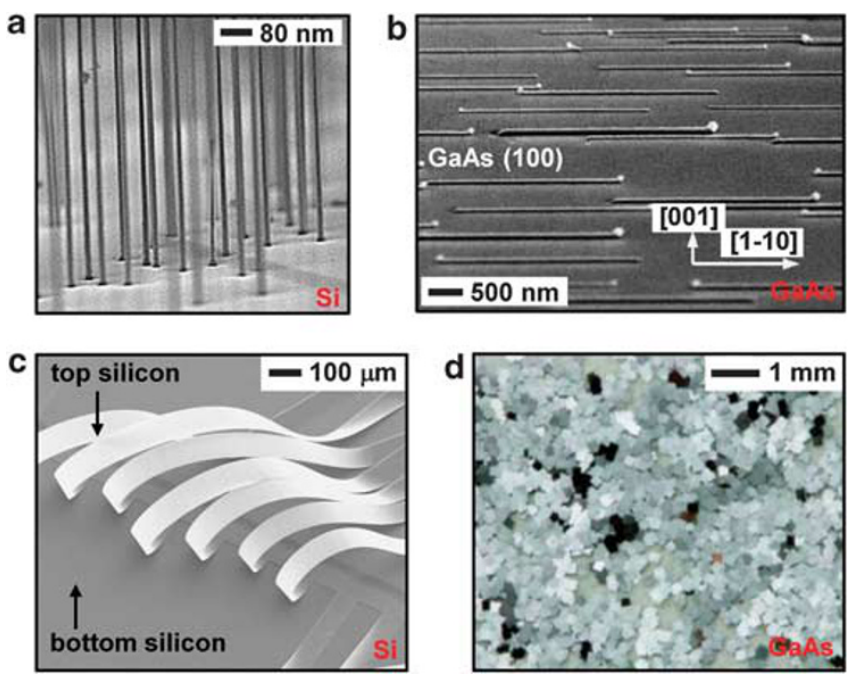

Figure 1 Single crystalline inorganic semiconductor nanomaterials formed by chemical synthesis and by lithographic processing. (a) Silicon NWs from chemical vapor deposition. Reprinted with permission from Goldberger et al. ${ }^{29}$ Copyright 2003 American Chemical Society. (b) (100) GaAs NWs horizontally grown on an (110) GaAs wafer. Reprinted with permission from Fortuna et al..$^{30}$ Copyright 2008 American Chemical Society. (c) Silicon nanoribbons released from an SOI wafer. (d) Collection of GaAs plates formed by selective wet etching of AIAs layers in a GaAs/AIAs multilayer stack. Reprinted with permission from Yoon et al. ${ }^{45}$ Copyright 2010 Nature Publishing Group.
Controlling the spatial layouts of large collections of individual NWs is critical for their practical use in electronic and optoelectronic devices. One approach is to pattern the metal catalysts, as a way to control NW dimensions, spacings and densities. ${ }^{27,28}$ Representative examples of vertically oriented NWs formed in this way appear in Figure $1 a^{29}$ Horizontally aligned arrays offer natural compatibility with established, planar device designs. Vertical arrays can be converted into horizontal ones by mechanical means, as described subsequently. ${ }^{34}$ Alternatively, control of epitaxial growth conditions can, in certain instances, yield this horizontal alignment directly in materials such as GaAs, where preferential interactions between NWs and a (100) GaAs growth substrate exist along the (110) direction during metal organic chemical vapor deposition. ${ }^{30}$ In other related approaches, vapor-liquid-solid (VLS) can lead to millimeter long and aligned horizontal GaN NWs on sapphire substrates, in mechanisms thought to be similar to those that yield guided growth of aligned single-wall carbon nanotubes along atomic steps of miscut sapphire. ${ }^{35,36}$ Horizontal but kinked or zigzag shapes in silicon NWs result from controlled formation of triangular joints between straight NWs. ${ }^{37}$ Several review articles provide comprehensive summaries of these and other approaches. $22-24,27,28,32,38,39$

More recent routes to NWs combine wafer-scale semiconductor growth with an advanced lithographic processing. ${ }^{27}$ An appealing feature of such strategies is their ability to control NW dimensions and layouts with engineering precision, in high quality materials with well-controlled distributions of dopants. Here, nanofabrication techniques based on conventional (for example, photolithography, electron beam lithography) or unconventional (for example, soft lithograpy, imprint lithography) methods define masks for wet or dry etching of a suitably prepared wafer. Removing embedded sacrificial layers, or eliminating underlying materials by isotropic or anisotropic etching, releases collections of NWs from the wafer surface and, in some instances, into its depth. ${ }^{40-46}$

Because lithography defines the lateral dimensions, these procedures can form not only NWs but also nanostructures with a variety of shapes and sizes-membranes, platelets, disks, bars and so on. Nanomembranes, in particular, are attractive because their twodimensional layouts match those of the most successful device geometries in electronics and optoelectronics. ${ }^{13}$ As a simple example, Si NMs can be formed by releasing the top Si layer of a silicon-on-insulator (SOI) substrate by etching away the buried oxide layer with hydrofluoric acid. ${ }^{47,48}$ Figure 1c shows narrow NMs of Si (100) created in this manner. Other SOI-like structures that can serve as routes to different nanomaterials include germanium-on-insulator, strained-SOI and silicon-germanium-on-insulator, as well as III-V semiconductors and many other combinations. ${ }^{46,49}$

A low cost alternative to SOI-derived Si NMs involves anisotropic etching of Si wafers with (111) crystallographic orientation. ${ }^{40,41}$ Here, reactive ion etching first defines vertical trenches in the wafer surface, oriented perpendicular to the (110) direction. Passivating the top surfaces and the sidewalls of the resulting relief, followed by anisotropic etching with potassium hydroxide or tetramethyl ammonium hydroxide undercuts the silicon beneath the raised regions by removing material along the (110) directions, to release Si NMs with thicknesses defined by the depths of the trenches. Related steps, implemented with trenches that have sculpted sidewalls, can yield bulk quantities of Si NMs from multilayer stacks in a single processing sequence. ${ }^{42}$ Other concepts use anisotropic etching of bulk wafers of GaAs and InP to yield NWs of these materials. ${ }^{43}$

Different approaches to compound semiconductor materials exploit epitaxial growth techniques to create multilayer assemblies 
with embedded layers that can be selectively removed as an extension of traditional epitaxial lift-off techniques. ${ }^{45}$ For example, alternating layers of GaAs and AlAs grown by metal organic chemical vapor deposition on a GaAs substrate yield a structure that can release large quantities of GaAs NMs upon elimination of the AlAs by etching in hydrofluoric acid. As with the cases described above, the resulting NMs have exceptionally well-controlled sizes, thicknesses and material quality (Figure 1d).

\section{ASSEMBLY}

Integrated systems that use NWs or NMs require their delivery onto substrates of interest at high throughput and with precise control over spatial position and orientation. Manipulating nanomaterials in this way is challenging owing to their small dimensions, mechanical fragility and tendency to aggregate and bond irreversibly to surfaces. ${ }^{50}$ Two conceptually different types of approaches have been explored extensively. The first, which we refer to as guided self-assembly, uses flow fields, ${ }^{51-56}$ electrical/magnetic forces, ${ }^{57-64}$ relief structures or other means 65 to guide the motion of nanomaterials in fluid suspensions before or during their delivery to a receiving substrate. One example exploits Langmuir-Blodgett techniques, in which NWs that float at a liquid-air interface organize into dense, aligned arrays through the action of uniaxial compressive forces applied to the surface ${ }^{51-56}$ (Figure 2a). A substrate, drawn from beneath the assembly affects transfer without substantially disrupting the orientational order. Flow-induced alignment can also be exploited in liquid
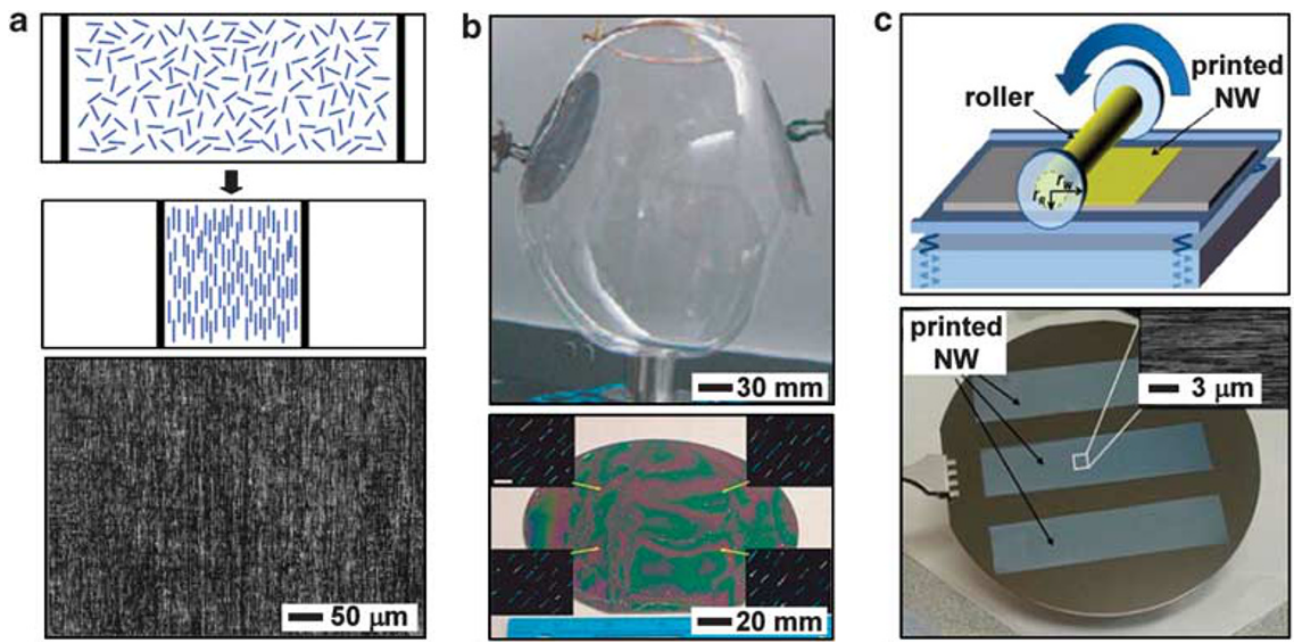

Figure 2 Three techniques for guided self-assembly of horizontally aligned arrays of synthesized NWs. (a) Schematic illustration of Langmuir-Blodgett assembly of NWs at a liquid-air interface (top), and image of aligned NWs (bottom). Reprinted with permission from Yang. ${ }^{51}$ Copyright 2003 Nature Publishing Group. (b) Inflated bubble of polymer with embedded NWs (top). Contacting a substrate transfers aligned NWs (bottom). The insets provide magnified views. Reprinted with permission from Yu et al. ${ }^{66}$ Copyright 2007 Nature Publishing Group. (c) Schematic illustration of dry contact transfer of NWs from a source to a receiving substrate in a roller press, by use of directional shear forces (top) and image of transferred NWs (bottom) on a wafer, with magnified views of representative regions. Reprinted with permission from Fan et al. ${ }^{68}$ Copyright 2009 WILEY-VCH.
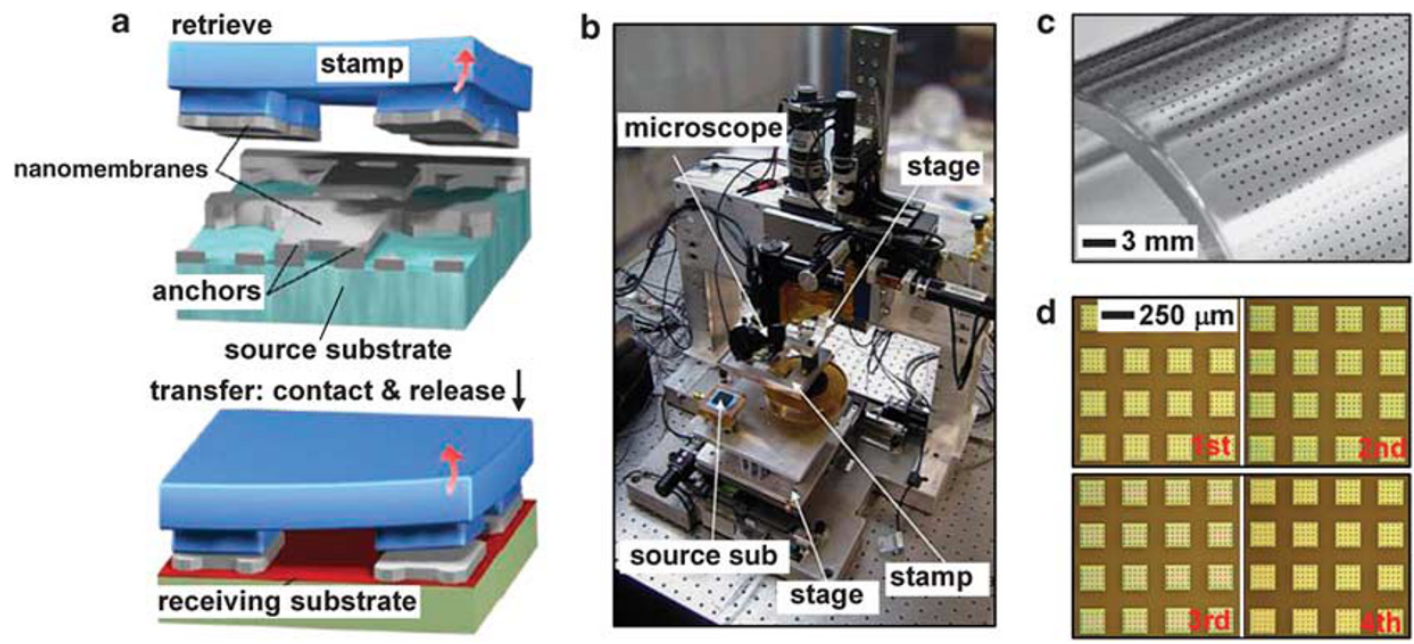

Figure 3 Transfer printing for deterministic assembly of nanomembranes formed by lithographic processing of a wafer substrate. (a) Schematic illustration of selective retrieval (top) and printing (bottom) of nanomembranes using a stamp. Grey, red, blue and yellowish green color represent inorganic nanomaterial, adhesive, stamp and receiving substrate, respectively. (b) An image of an automated printer that consists of a programmable, three axis translation stage, a stamp fixture, a high resolution microscope and integrated load cells. (c) GaAs nanomembranes printed in a sparse array on a plastic sheet, subsequently bent around a glass cylinder (top). (d) Magnified view of printed GaAs nanomembranes derived from different layers of epitaxial growth (bottom). Reprinted with permission from Yoon et al. ${ }^{45}$ Copyright 2010 Nature Publishing Group. 
and polymer suspensions of NWs by using microfluidic channels and inflating bubbles, respectively (Figure 2b). ${ }^{66,67}$ In both cases, shear stresses create a tendency for the NWs to align along the flow direction. Transfer occurs upon sedimentation or physical transfer to a receiver substrate, as shown in the case of the polymer approach in the bottom frame of Figure 2b. Although these and other guided assembly techniques have some promise, their current capabilities do not allow the areal densities, uniformity in coverage or levels of control over orientation and end-to-end registry needed to address the sorts of applications envisioned here.

Dry assembly processes overcome some of these limitations, with the additional ability to be implemented in continuous, roll-to-roll modes over large areas (Figure 2c). ${ }^{68}$ Here, the starting point is the growth of vertically aligned arrays of NWs, using techniques such as those described previously. ${ }^{68}$ Physical contact with a receiving substrate while applying a controlled shear force results in the transfer of horizontally aligned arrays. A representative example appears in the bottom frame of Figure 2c. The inset shows a magnified view of some NWs. The principle disadvantage of this process is that it involves NWs whose electronic properties, dimensions and orientational order are poorly controlled, in a comparative sense to nanostructures formed using wafer-based schemes.

Transfer printing techniques that use soft, elastomeric stamps in conjunction with nanomaterials formed by lithographic processes eliminate the disadvantages highlighted above to offer capabilities that meet requirements for integration into realistic devices. The key attributes are deterministic control both at the level of the nanomaterials and their assembly into desired configurations. In an example of this process, (Figure 3a) NMs (or NWs) are prepared on and anchored to source substrates in a way that retains their lithographically defined locations even after complete undercut etching. ${ }^{45,69,70}$ Contact of an elastomeric stamp with matching features of relief on its surface leads to van der Waals forces that can be sufficiently large to break these anchors and lift selected collections of NMs onto the surface of the stamp when it is peeled away. ${ }^{45,69,70}$ A stamp, inked in this manner, can deliver the NMs, in a single impression, to a substrate of interest. The mechanically soft nature of the stamp avoids fracture in the NMs during this process. ${ }^{45,69,71-74}$

An important consideration is in control over the adhesion between nanomaterials and the surfaces of the stamps, in ways that allow switching from strong to weak states for retrieval and printing, respectively. ${ }^{69,70,75,76}$ Various approaches, ranging from those that exploit viscoelastic effects ${ }^{69,75}$ to interface shear loading ${ }^{76}$ to pressureinduced contact modulation, ${ }^{70,77}$ can be effective. In many cases, separate adhesive layers on the receiving substrate can further facilitate the printing. When implemented with automated tools, (Figure 3b) these concepts can offer yields exceeding 99.9\%, with submicron registration accuracy and throughputs of up to one million NMs per hour (depending on the layouts and the sizes of the stamps). ${ }^{45,71}$ Examples of arrays of GaAs NMs derived through lift-off from a source wafer according to procedures described previously, and then transfer printed onto a flexible substrate appear in Figures $3 \mathrm{c}$ and $\mathrm{d}^{45}$

\section{DEVICES}

Organized collections of inorganic semiconductor nanomaterials on plastic sheets or rubber membranes can be used as active components for diverse types of high performance electronic (Figure $4 a)^{78}$ and optoelectronic devices (Figures $4 \mathrm{~b}$ and $\mathrm{c})^{79}$ that have the ability to flex and stretch, even to large levels of deformation, with linear elastic responses. On comparison with related systems that rely on organic
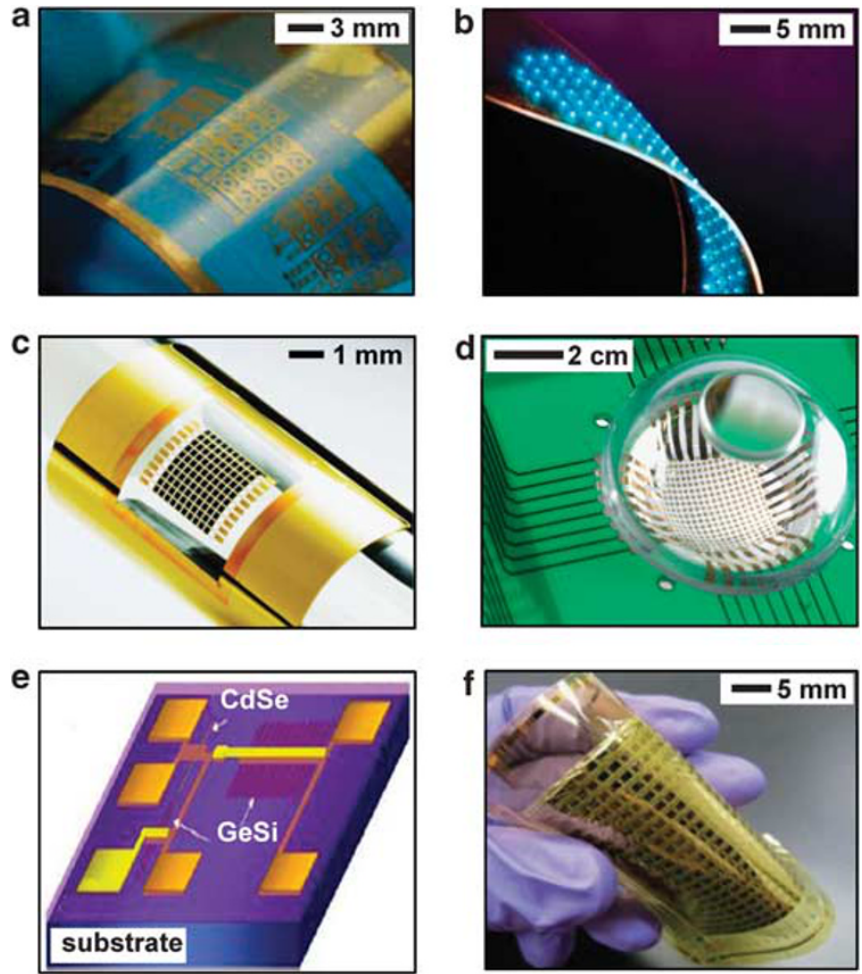

Figure 4 Flexible and stretchable electronic and optoelectronic devices. (a) Single crystal silicon transistors and wireless communication components on a flexible substrate. Reprinted with permission from Fan et al. ${ }^{68}$ Copyright 2010 WILEY-VCH. (b) Array of microscale, InGaN LEDs on a plastic sheet. Reprinted with permission from Kim et al. ${ }^{79}$ Copyright 2011 National Academy of Sciences. (c) High efficiency GaAs photovoltaic cells on a flexible substrate wrapped around a glass cylinder. Reprinted with permission from Yoon et al. ${ }^{45}$ Copyright 2010 Nature Publishing Group. (d) Hemispherical photodiode array for an electronic eye camera. Reprinted with permission from Ko et al. ${ }^{80}$ Copyright 2008 Nature Publishing Group. (e) Multiplexed photodetector array using $\mathrm{CdSe}$ and $\mathrm{Ge} / \mathrm{Si} \mathrm{NWs}$ for optoelectronic and electronic components, respectively. Reprinted with permission from Fan et al. ${ }^{81}$ Copyright 2008 National Academy of Sciences. (f) Pressure sensor array as a touch sensor, using nanowire multiplexing circuits. Reprinted with permission from Takei et al. ${ }^{82}$ Copyright 2010 Nature Publishing Group.

semiconductors, similar or better mechanical characteristics (for example, degree of bendability) can be achieved, but with far superior operational features because of the use of high performance materials, such as GaN for LEDs or high electron mobility transistors, Si for metal oxide semiconductor field effect transistors and GaAs for solar cells and photodetectors. A primary appeal is the ability to provide function that cannot be reproduced using conventional wafer-based technologies. As an example, Figure $4 \mathrm{~d}$ shows an electronic eyeball camera, inspired by the curvilinear shape and geometry of the mammalian retina. ${ }^{80}$ Here, printed Si NMs, interconnected into stretchable configurations on rubber substrates, yield multiplexed photodetector arrays. Fabrication occurs in planar layouts that are then deformed geometrically into hemispherical shapes by conformal wrapping onto a suitable, curved surface. The advantage of curvilinear layouts is that they can be selected to match, precisely, the intrinsic curvature of images that form with simple lenses. The result is reduced aberrations, expanded fields of view and improved uniformity in illumination compared 
with planar devices when similarly simple lenses (that is, small, lightweight, low cost) are used. Besides Si NMs, photodetectors containing vertically grown arrays of CdSe and Ge/Si NWs transferred onto a flexible substrate can serve as building blocks for imaging arrays that can be bent into conical or cylindrical forms (Figure 4e). ${ }^{81}$ Related systems exploiting arrays of inorganic Si NWs for measuring pressure are also possible (Figure $4 \mathrm{f}$ ). ${ }^{82}$

The ability to mimic biology in device designs immediately suggests opportunities to integrate with biology. Advanced tools for biomedicine are possible using concepts similar to those of Figure $4 \mathrm{~d}$, but with multiple sensing and electronic modalities, and the capacity to laminate onto soft, moist curvilinear tissues with robust adhesion, waterproof construction and without any significant effects of mass loading or mechanical constraint (Figure $5 \mathrm{a}$ ). One of the first devices of this type used an interconnected, dense array of 288 active electrodes ${ }^{83}$ based on Si NM electronics, with each node consisting of a contact pad interface with tissue, an amplifier and a multiplexer. The result is a sensor sheet with high speed capabilities in isochronal mapping of electrical potential (that is, electrophysiology) to yield real-time visualization of the origins of depolarization waves on the surface of the heart (Figure 5b). The multiplexing scheme allows the use of only 36 wires to address 288 individual electrodes with sufficiently fast sampling speeds. A notable feature of operation is that capillary interactions at the moist tissue surface are sufficient to hold this device in place even during vigorous beating of the heart. Analogous systems can perform mapping of the brain, also with unmatched resolution. Figure $5 c$ shows a representative device consisting of 360 active electrode channels in contact with the visual cortical surface of a live feline model. ${ }^{84}$ As with the cardiac system, high-speed multiplexing circuits based on Si NM metal oxide semiconductor field effect transistors enable mapping of excitation patterns over large surfaces with high sampling rates $(>10 \mathrm{ksps}$ per electrode; Figure 5d) and with far fewer external wire connections than devices that demand direct addressing. As an important demonstration of this technology, electrical activity was mapped during local administration of seizure-inducing drugs in a live animal. ${ }^{84}$ The data reveal complex waves of depolarization, in the form of spiral clockwise and counter-clockwise disturbances during local seizure episodes. These results collectively represent the first observation of such phenomena in living heart and brain tissues. At the cellular and subcellular levels, nanowire assemblies and transistors hold additional promise for recording electrical potentials from inside of individual cells or for detecting and characterizing DNA. ${ }^{85-87}$

Clinical utility in many applications, such as mapping of cardiac electrophysiology, is highest for devices that can be deployed in slender, mechanically flexible and minimally invasive formats. Balloon catheters represent an attractive platform for delivering into the body stretchable versions of electronics that have functionality similar to the flexible devices of Figure $5 .^{88}$ Figure $6 \mathrm{a}$ shows an example, in deflated (top) and inflated (bottom) states, of a device that contains a collection of electrodes, temperature sensors and microscale LEDs. Here, components for both sensing and affecting therapies mount on the balloon surface, in an open mesh configuration with filamentary serpentine interconnects, to allow large-scale stretching associated with inflation, where strains can exceed $200 \%$ in the latitudinal and longitudinal directions. A representative electrocardiogram recording from the right ventricle of a swine model appears in Figure $6 \mathrm{~b}$, collected during full inflation of the balloon. In addition to electrodes for electrophysiology sensing and radio frequency ablation, temperature sensors and LEDs, the same system incorporates sensors for flow
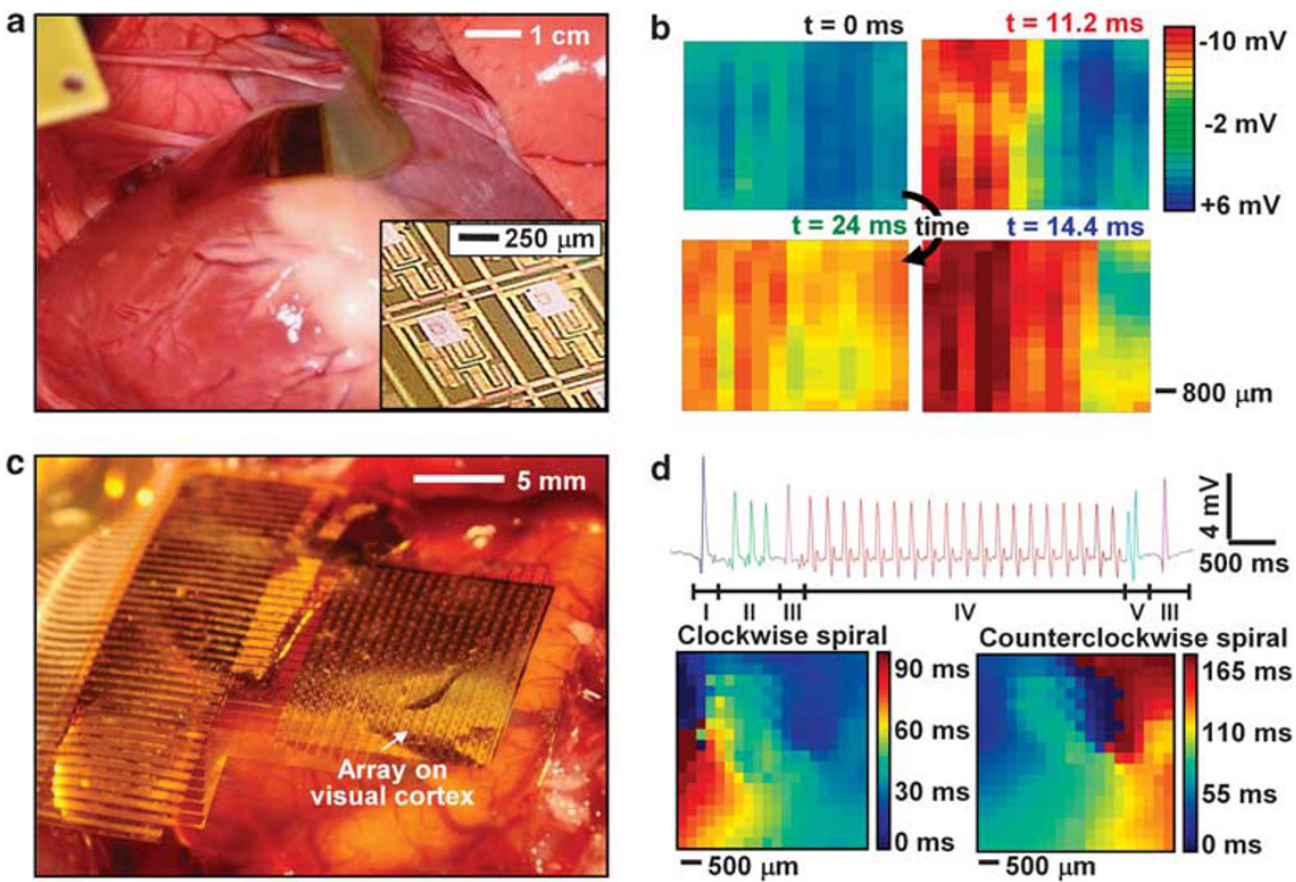

Figure 5 Flexible electronics in biomedical devices. (a) Flexible, actively multiplexed and amplified electrode array conformally wrapped on and adhered to the epicardial surface of a porcine animal model. The inset shows a magnified view of a single unit cell in the array. (b) Maps of electrocardiogram propagation waves, at various time intervals. Reprinted with permission from Viventi et al. ${ }^{83}$ Copyright 2010 American Association for the Advancement of Science. (c) High density system for mapping surface potentials on the brain, illustrated here on the visual cortex of a feline model. (d) Comparison of mapping results from a conventional single channel electrode (top) and from a high density neural sensor array (bottom), obtained during an induced seizure. High resolution maps show clockwise and counter-clockwise spiral patterns unseen with conventional electrodes. Reprinted with permission from Viventi et al. ${ }^{84}$ Copyright 2011 Nature Publishing Group. 


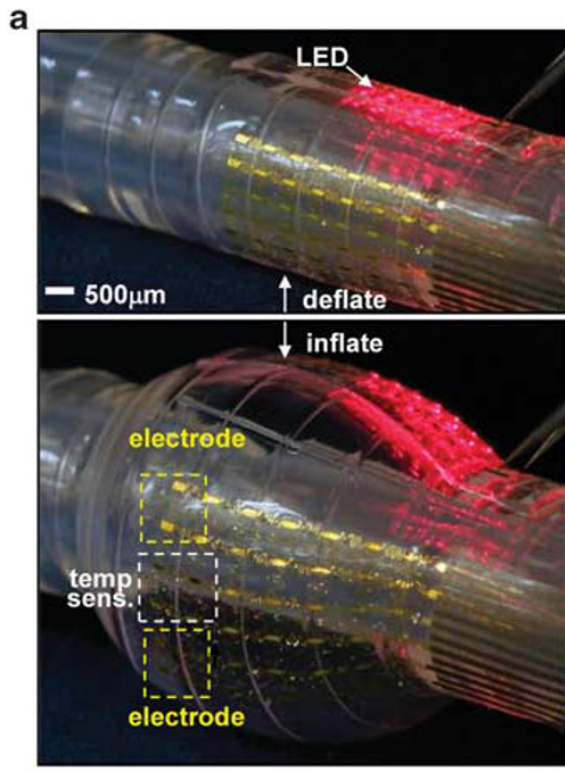

b

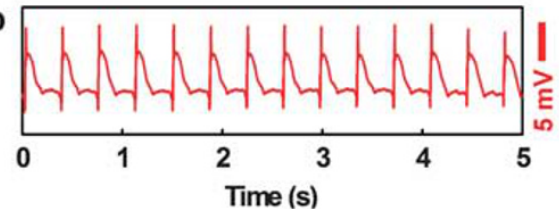

c

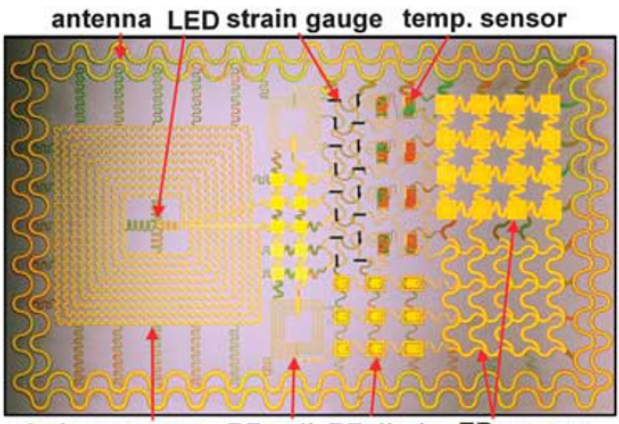

wireless power RF coil RF diode EP sensor
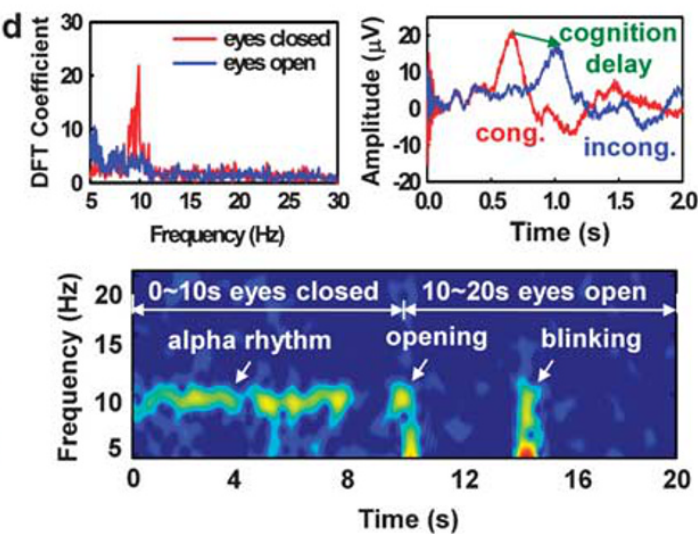

Figure 6 Stretchable electronics in biomedical devices. (a) Multifunctional sensor array on an inflatable balloon catheter for minimally invasive cardiac electrophysiology, shown in its deflated (top) and inflated (bottom) state. (b) Electrocardiogram mapping result from the right ventricle of a rabbit model. Reprinted with permission from Kim et al. ${ }^{88}$ Copyright 2011 Nature Publishing Group. (c) Multifunctional epidermal electronic system for physiological and electrophysiological monitoring. (d) Electroencephalogram monitoring of a $\sim 10-\mathrm{Hz}$ alpha rhythm with the patient's eyes closed and open (top left) and its spectrogram (bottom). Top right frame shows electroencephalogram recording of Stroop test, in which a cognition delay can be observed for incongruent cases. Reprinted with permission from Kim et al. ${ }^{89}$ Copyright 2011 American Association for the Advancement of Science.

and contact pressure. The multimodal operation of this instrumented balloon catheter can enhance the efficacy as well as decrease the time and cost associated with cardiac ablation procedures currently used to treat various forms of arrhythmias.

Extended versions of the design principles used in the systems of Figure 6a can enable classes of devices with physical properties (that is, modulus, thickness and mass density) that match those of the epidermis, for skin-mounted applications in both healthcare and nonhealthcare modes of use. ${ }^{89}$ The ability of such epidermal electronic devices to achieve intimate contact by simple lamination onto the skin yields a means for integration that is mechanically invisible to the user. This coupling also provides an effective electrical interface, both for measurement of electrical, chemical, mechanical activity and for stimulation through the skin. Figure $6 \mathrm{c}$ shows a demonstration platform for a broad collection of passive and active elements, from sensors of temperature, strain and electrical activity to Si NM metal oxide semiconductor field effect transistors and diodes, to inductors, capacitors and radio frequency antennas. Figure $6 \mathrm{~d}$ presents an example of electroencephalogram monitoring with this type of device during the closing and opening of the eyes with corresponding spectrogram data (bottom). The results suggest potential use in cognitive state monitors and brain-computer interfaces.

As highlighted in this review, advanced concepts in semiconductor nanomaterials, assembly methods and mechanics designs now establish realistic foundations for technologies with important capabilities that are different than and complementary to those of traditional, wafer-based systems. Some of the most promising areas are in bio-inspired device design and bio-integrated systems. For the latter, opportunities for creative engineering exist in integrated power supply and wireless communications. Key scientific issues relate to the range of possible interactions between these components and the surrounding biology. The range of interesting topics in basic and applied research create a fertile area for future work, where successful outcomes can provide broad, societal benefits.

\section{ACKNOWLEDGEMENTS}

This study is supported by the National Science Foundation under Grant DMI-0328162 and the US Department of Energy, Division of Materials Sciences under Award No. DE-FG02-07ER46471 through the Materials Research Laboratory and Center for Microanalysis of Materials (DE-FG0207ER46453) at the University of Illinois at Urbana-Champaign. JAR acknowledges a National Security Science and Engineering Faculty Fellowship. This work was also supported by the Global Frontier Research Center for Advanced Soft Electronics.

1 Stucky, G. \& Macdougall, J. Quantum confinement and host guest chemistry - probing a new dimension. Science 247, 669-678 (1990).

2 Goldstein, A., Echer, C. \& Alivisatos, A. Melting in semiconductor nanocrystals. Science 256, 1425-1427 (1992).

3 Kratschmer, W., Lamb, L., Fostiropoulos, K. \& Huffman, D. Solid C-60 - a new form of carbon. Nature 347, 354-358 (1990).

4 Roduner, E. Size matters: why nanomaterials are different. Chem. Soc. Rev. 35, 583592 (2006).

5 Yin, Y. \& Alivisatos, A. Colloidal nanocrystal synthesis and the organic-inorganic interface. Nature 437, 664-670 (2005). 
6 Lieber, C. M. Semiconductor nanowires: a platform for nanoscience and nanotechnology. MRS Bull. 36, 1052-1063 (2011).

7 Ithurria, S., Tessier, M. D., Mahler, B., Lobo, R. P. S. M., Dubertret, B. \& Efros, A. L. Colloidal nanoplatelets with two-dimensional electronic structure. Nat. Mater. 10, 936-941 (2011).

8 Whitesides, G., Mathias, J. \& Seto, C. Molecular self-assembly and nanochemistry - a chemical strategy for the synthesis of nanostructures. Science 254, 1312-1319 (1991).

9 Velev, O. Self-assembly of unusual nanoparticle crystals. Science 312, 376-377 (2006).

10 Liu, K., Zhao, N. \& Kumacheva, E. Self-assembly of inorganic nanorods. Chem. Soc. Rev. 40, 656-671 (2011).

11 Baca, A. J., Ahn, J., Sun, Y., Meitl, M. A., Menard, E., Kim, H., Choi, W. M., Kim, D. Huang, Y. \& Rogers, J. A. Semiconductor wires and ribbons for high-performance flexible electronics. Angew. Chem. Int. Edit. 47, 5524-5542 (2008).

12 Sun, Y. \& Rogers, J. A. Inorganic semiconductors for flexible electronics. Adv. Mater. 19, 1897-1916 (2007).

13 Rogers, J. A., Lagally, M. G. \& Nuzzo, R. G. Synthesis, assembly and applications of semiconductor nanomembranes. Nature 477, 45-53 (2011)

$14 \mathrm{Lu}$, W. \& Lieber, C. M. Nanoelectronics from the bottom up. Nat. Mater. 6, 841-850 (2007).

$15 \mathrm{Kim}$, D., Xiao, J., Song, J., Huang, Y. \& Rogers, J. A. Stretchable, curvilinear electronics based on inorganic materials. Adv. Mater. 22, 2108-2124 (2010).

16 Rogers, J. A., Someya, T. \& Huang, Y. Materials and mechanics for stretchable electronics. Science 327, 1603-1607 (2010).

17 Park, S., Ahn, J., Feng, X., Wang, S., Huang, Y. \& Rogers, J. A. Theoretical and experimental studies of bending of inorganic electronic materials on plastic substrates. Adv. Funct. Mater. 18, 2673-2684 (2008).

18 Reuss, R., Chalamala, B., Moussessian, A., Kane, M., Kumar, A., Zhang, D., Rogers, J., Hatalis, M., Temple, D., Moddel, G., Eliasson, B., Estes, M., Kunze, J., Handy, E., Harmon, E., Salzman, D., Woodall, J., Alam, M., Murthy, J., Jacobsen, S., Olivier, M. Markus, D., Campbell, P. \& Snow, E. Macroelectronics: perspectives on technology and applications. Proc. IEEE 93, 1239-1256 (2005).

19 Reuss, R., Hopper, D. \& Park, J. Macroelectronics. MRS Bull. 31, 447-450 (2006).

20 Timko, B. P., Cohen-Karni, T., Qing, Q., Tian, B. \& Lieber, C. M. Design and implementation of functional nanoelectronic interfaces with biomolecules, cells, and tissue using nanowire device arrays. IEEE T. Nanotechnol. 9, 269-280 (2010).

$21 \mathrm{Kim}$, D., Lu, N., Huang, Y. \& Rogers, J. A. Materials for stretchable electronics in bioinspired and biointegrated devices. MRS Bull. doi:10.1557/mrs.2012.36 in press.

22 Li, Y., Qian, F., Xiang, J. \& Lieber, C. M. Nanowire electronic and optoelectronic devices. Mater. Today 9, 18-27 (2006).

23 Lieber, C. M. \& Wang, Z. L. Functional nanowires. MRS Bull. 32, 99-108 (2007).

24 Patolsky, F., Timko, B. P., Zheng, G. \& Lieber, C. M. Nanowire-based nanoelectronic devices in the life sciences. MRS Bull. 32, 142-149 (2007).

25 Xiao, J., Ryu, S. Y., Huang, Y., Hwang, K., Paik, U. \& Rogers, J. A. Mechanics of nanowire/nanotube in-surface buckling on elastomeric substrates. Nanotechnology 21, 085708 (2010).

26 Wagner, R. \& Ellis, W. Vapor-liquid-solid mechanism of single crystal growth. Appl. Phys. Lett. 4, 89-90 (1964).

27 Fan, H., Werner, P. \& Zacharias, M. Semiconductor nanowires: from self-organization to patterned growth. Small 2, 700-717 (2006).

28 Schmidt, V., Wittemann, J. V. \& Goesele, U. Growth, thermodynamics, and electrical properties of silicon nanowires. Chem. Rev. 110, 361-388 (2010).

29 Goldberger, J., Hochbaum, A. I., Fan, R. \& Yang, P. Silicon vertically integrated nanowire field effect transistors. Nano Lett. 6, 973-977 (2006).

30 Fortuna, S. A., Wen, J., Chun, I. S. \& Li, X. Planar GaAs nanowires on GaAs (100) substrates: self-aligned, nearly twin-defect free, and transfer-printable. Nano Lett. 8, 4421-4427 (2008).

31 Yan, R., Gargas, D. \& Yang, P. Nanowire photonics. Nat. Photonics 3, 569-576 (2009).

32 Hochbaum, A. I. \& Yang, P. Semiconductor nanowires for energy conversion. Chem. Rev. 110, 527-546 (2010).

33 Garnett, E. C., Brongersma, M. L., Cui, Y. \& McGehee, M. D. Nanowire solar cells. Ann. Rev. Mater. Res. 41, 269-295 (2011).

34 Fan, Z., Ho, J. C., Takahashi, T., Yerushalmi, R., Takei, K., Ford, A. C., Chueh, Y. \& Javey, A. Toward the development of printable nanowire electronics and sensors. Adv. Mater. 21, 3730-3743 (2009).

35 Kang, S. J., Kocabas, C., Ozel, T., Shim, M., Pimparkar, N., Alam, M. A., Rotkin, S. V. \& Rogers, J. A. High-performance electronics using dense, perfectly aligned arrays of single-walled carbon nanotubes.. Nat. Nanotechnol. 2, 230-236 (2007).

36 Tsivion, D., Schvartzman, M., Popovitz-Biro, R., von Huth, P. \& Joselevich, E. Guided growth of millimeter-long horizontal nanowires with controlled orientations. Science 333, 1003-1007 (2011)

37 Tian, B., Xie, P., Kempa, T. J., Bell, D. C. \& Lieber, C. M. Single-crystalline kinked emiconductor nanowire superstructures. Nat. Nanotechnol. 4, 824-829 (2009).

38 Hayden, O., Agarwal, R. \& Lu, W. Semiconductor nanowire devices. Nano Today 3, 12 22 (2008).

39 Zhang, W. \& Yang, S. In situ fabrication of inorganic nanowire arrays grown from and aligned on metal substrates. Acc. Chem. Res. 42, 1617-1627 (2009).

40 Mack, S., Meitl, M., Baca, A., Zhu, Z. \& Rogers, J. Mechanically flexible thin-film transistors that use ultrathin ribbons of silicon derived from bulk wafers. Appl. Phys. Lett. 88, 213101 (2006).
41 Baca, A. J., Meitl, M. A., Ko, H. C., Mack, S., Kim, H., Dong, J., Ferreira, P. M. \& Rogers, J. A. Printable single-crystal silicon micro/nanoscale ribbons, platelets and bars generated from bulk wafers. Adv. Funct. Mater. 17, 3051-3062 (2007).

42 Ko, H. C., Baca, A. J. \& Rogers, J. A. Bulk quantities of single-crystal silicon micro-/ nanoribbons generated from bulk wafers. Nano Lett. 6, 2318-2324 (2006).

43 Sun, Y., Khang, D., Hua, F., Hurley, K., Nuzzo, R. \& Rogers, J. Photolithographic route to the fabrication of micro/nanowires of III-V semiconductors. Adv. Funct. Mater. 15 30-40 (2005).

44 Garnett, E. \& Yang, P. Light trapping in silicon nanowire solar cells. Nano Lett. 10, 1082-1087 (2010).

45 Yoon, J., Jo, S., Chun, I. S., Jung, I., Kim, H., Meitl, M., Menard, E., Li, X., Coleman, J. J., Paik, U. \& Rogers, J. A. GaAs photovoltaics and optoelectronics using releasable multilayer epitaxial assemblies. Nature 465, 329-333 (2010).

$46 \mathrm{Li}, \mathrm{X}$. Strain induced semiconductor nanotubes: from formation process to device applications. J. Phys. D Appl. Phys. 41, 193001 (2008).

47 Ahn, J., Kim, H., Lee, K. J., Zhu, Z., Menard, E., Nuzzo, R. G. \& Rogers, J. A. Highspeed mechanically flexible single-crystal silicon thin-film transistors on plastic substrates. IEEE Electron. Device Lett. 27, 460-462 (2006).

48 Service, R. Materials science - inorganic electronics begin to flex their muscle. Science 312, 1593-1594 (2006).

49 Cho, A. Nanotechnology - pretty as you please, curling films turn themselves into nanodevices. Science 313, 164-165 (2006).

50 Service, R. Assembling nanocircuits from the bottom up. Science 293, 782-785 (2001).

51 Yang, P. Wires on water. Nature 425, 243-244 (2003).

52 Whang, D., Jin, S., Wu, Y. \& Lieber, C. Large-scale hierarchical organization of nanowire arrays for integrated nanosystems. Nano Lett. 3, 1255-1259 (2003).

53 Tao, A., Kim, F., Hess, C., Goldberger, J., He, R., Sun, Y., Xia, Y. \& Yang, P. Langmuirblodgett silver nanowire monolayers for molecular sensing using surface-enhanced raman spectroscopy. Nano Lett. 3, 1229-1233 (2003).

54 Wang, D., Chang, Y., Liu, Z. \& Dai, H. Oxidation resistant germanium nanowires: bulk synthesis, long chain alkanethiol functionalization, and langmuir-blodgett assembly. J. Am. Chem. Soc. 127, 11871-11875 (2005)

55 Acharya, S., Panda, A., Belman, N., Efrima, S. \& Golan, Y. A semiconductor-nanowire assembly of ultrahigh junction density by the langmuir-blodgett technique. Adv. Mater. 18, 210-213 (2006).

56 Park, J., Shin, G. \& Ha, J. S. Controlling orientation of $\mathrm{V}_{2} \mathrm{O}_{5}$ nanowires within micropatterns via microcontact printing combined with the gluing langmuir-blodgett technique. Nanotechnology 19, 395303 (2008).

57 Tanase, M., Silevitch, D., Hultgren, A., Bauer, L., Searson, P., Meyer, G. \& Reich, D. Magnetic trapping and self-assembly of multicomponent nanowires. J. Appl. Phys. 91, 8549-8551 (2002).

58 Smith, P., Nordquist, C., Jackson, T., Mayer, T., Martin, B., Mbindyo, J. \& Mallouk, T. Electric-field assisted assembly and alignment of metallic nanowires. Appl. Phys. Lett. 77, 1399-1401 (2000).

59 Boote, J. \& Evans, S. Dielectrophoretic manipulation and electrical characterization of gold nanowires. Nanotechnology 16, 1500-1505 (2005).

60 Hamers, R., Beck, J., Eriksson, M., Li, B., Marcus, M., Shang, L., Simmons, J. \& Streifer, J. Electrically directed assembly and detection of nanowire bridges in aqueous media. Nanotechnology 17, S280-S286 (2006).

61 Vijayaraghavan, A., Blatt, S., Weissenberger, D., Oron-Carl, M., Hennrich, F., Gerthsen, D., Hahn, H. \& Krupke, R. Ultra-large-scale directed assembly of single-walled carbon nanotube devices. Nano Lett. 7, 1556-1560 (2007).

62 Fan, D. L., Cammarata, R. C. \& Chien, C. L. Precision transport and assembling of nanowires in suspension by electric fields. Appl. Phys. Lett. 92, 093115 (2008).

63 Raychaudhuri, S., Dayeh, S. A., Wang, D. \& Yu, E. T. Precise semiconductor nanowire placement through dielectrophoresis. Nano Lett. 9, 2260-2266 (2009).

64 Freer, E. M., Grachev, O., Duan, X., Martin, S. \& Stumbo, D. P. High-yield self-limiting single-nanowire assembly with dielectrophoresis. Nat. Nanotechnol. 5, 525-530 (2010).

65 Xia, Y., Yang, P., Sun, Y., Wu, Y., Mayers, B., Gates, B., Yin, Y., Kim, F. \& Yan, Y. Onedimensional nanostructures: synthesis, characterization, and applications. Adv. Mater. 15, 353-389 (2003)

66 Yu, G., Cao, A. \& Lieber, C. M. Large-area blown bubble films of aligned nanowires and carbon nanotubes.. Nat. Nanotechnol. 2, 372-377 (2007)

67 Yu, G., Li, X., Lieber, C. M. \& Cao, A. Nanomaterial-incorporated blown bubble films for large-area, aligned nanostructures. J. Mater. Chem. 18, 728-734 (2008).

68 Fan, Z., Ho, J. C., Takahashi, T., Yerushalmi, R., Takei, K., Ford, A. C., Chueh, Y.-L. \& Javey, A. Toward the development of printable nanowire electronics and sensors. Adv. Mater. 21, 3730-3743 (2009).

69 Meitl, M., Zhu, Z., Kumar, V., Lee, K., Feng, X., Huang, Y., Adesida, I., Nuzzo, R. \& Rogers, J. Transfer printing by kinetic control of adhesion to an elastomeric stamp. Nat. Mater. 5, 33-38 (2006).

70 Kim, S., Wu, J., Carlson, A., Jin, S. H., Kovalsky, A., Glass, P., Liu, Z., Ahmed, N., Elgan, S. L., Chen, W., Ferreira, P. M., Sitti, M., Huang, Y. \& Rogers, J. A. Microstructured elastomeric surfaces with reversible adhesion and examples of their use in deterministic assembly by transfer printing. Proc. Natl. Acad. Sci. USA 107, 17095-17100 (2010)

71 Chanda, D., Shigeta, K., Gupta, S., Cain, T., Carlson, A., Mihi, A., Baca, A. J., Bogart, G. R., Braun, P. \& Rogers, J. A. Large-area flexible 3D optical negative index metamaterial formed by nanotransfer printing. Nat. Nanotechnol. 6, 402-407 (2011).

72 Lee, K., Motala, M., Meitl, M., Childs, W., Menard, E., Shim, A., Rogers, J. \& Nuzzo, R. Large-area, selective transfer of microstructured silicon: a printing-based approach to 
high-performance thin-film transistors supported on flexible substrates. Adv. Mater. 17, 2332-2336 (2005).

73 Menard, E., Lee, K., Khang, D., Nuzzo, R. \& Rogers, J. A printable form of silicon for high performance thin film transistors on plastic substrates. Appl. Phys. Lett. 84 5398-5400 (2004).

74 Menard, E., Nuzzo, R. \& Rogers, J. Bendable single crystal silicon thin film transistors formed by printing on plastic substrates. Appl. Phys. Lett. 86, 093507 (2005).

75 Feng, X., Meitl, M. A., Bowen, A. M., Huang, Y., Nuzzo, R. G. \& Rogers, J. A. Competing fracture in kinetically controlled transfer printing. Langmuir 23, 1255512560 (2007).

76 Carlson, A., Kim-Lee, H., Wu, J., Elvikis, P., Cheng, H., Kovalsky, A., Elgan, S., Yu, Q. Ferreira, P. M., Huang, Y., Turner, K. T. \& Rogers, J. A. Shear-enhanced adhesiveless transfer printing for use in deterministic materials assembly. Appl. Phys. Lett. 98, 264104 (2011).

77 Wu, J., Kim, S., Carlson, A., Lu, C. F., Hwang, K.-C., Huang, Y. \& Rogers, J. A. Contact radius of stamps in reversible adhesion. Theor. Appl. Mec. Lett. 1, 011001 (2011).

78 Sun, L., Qin, G., Seo, J., Celler, G. K., Zhou, W. \& Ma, Z. 12-GHz thin-film transistors on transferrable silicon nanomembranes for high-performance flexible electronics. Small 6, 2553-2557 (2010).

79 Kim, H., Brueckner, E., Song, J., Li, Y., Kim, S., Lu, C., Sulkin, J., Choquette, K., Huang, Y., Nuzzo, R. G. \& Rogers, J. A. Unusual strategies for using indium gallium nitride grown on silicon (111) for solid-state lighting. Proc. Natl. Acad. Sci. USA 108, 10072-10077 (2011).

80 Ko, H. C., Stoykovich, M. P., Song, J., Malyarchuk, V., Choi, W. M., Yu, C. Geddes,Joseph, III B., Xiao, J., Wang, S., Huang, Y. \& Rogers, J. A. A hemispherical electronic eye camera based on compressible silicon optoelectronics. Nature 454 748-753 (2008).

81 Fan, Z., Ho, J. C., Jacobson, Z. A., Razavi, H. \& Javey, A. Large-scale, heterogeneous integration of nanowire arrays for image sensor circuitry. Proc. Natl. Acad. Sci. USA 105, 11066-11070 (2008).

82 Takei, K., Takahashi, T., Ho, J. C., Ko, H., Gillies, A. G., Leu, P. W., Fearing, R. S. \& Javey, A. Nanowire active-matrix circuitry for low-voltage macroscale artificial skin. Nat. Mater. 9, 821-826 (2010).

83 Viventi, J., Kim, D., Moss, J. D., Kim, Y., Blanco, J. A., Annetta, N., Hicks, A., Xiao, J. Huang, Y., Callans, D. J., Rogers, J. A. \& Litt, B. A conformal, bio-interfaced class of silicon electronics for mapping cardiac electrophysiology. Sci. Transl. Med. 2, 24 ra22 (2010).

84 Viventi, J., Kim, D., Vigeland, L., Frechette, E. S., Blanco, J. A., Kim, Y., Avrin, A. E., Tiruvadi, V. R., Hwang, S., Vanleer, A. C., Wulsin, D. F., Davis, K., Gelber, C. E., Palmer, L., Van der Spiegel, J., Wu, J., Xiao, J., Huang, Y., Contreras, D., Rogers, J. A. \& Litt, B. Flexible, foldable, actively multiplexed, high-density electrode array for mapping brain activity in vivo. Nat. Neurosci. 14, 1599-1605 (2011).

85 Tian, B., Cohen-Karni, T., Qing, Q., Duan, X., Xie, P. \& Lieber, C. M. Three-dimensional, flexible nanoscale field-effect transistors as localized bioprobes. Science $\mathbf{3 2 9}, 830$ 834 (2010).

86 Duan, X., Gao, R., Xie, P., Cohen-Karni, T., Qing, Q., Choe, H., Tian, B., Jiang, X. \& Lieber, C. Intracellular recordings of action potentials by an extracellular nanoscale field-effect transistor. Nat. Nanotechnol. 7, 174-179 (2012).

87 Xie, P., Xiong, Q., Fang, Y., Qing, Q. \& Lieber, C. M. Local electrical potential detection of DNA by nanowire-nanopore sensors. Nat. Nanotechnol. 7, 119-125 (2012).

88 Kim, D., Lu, N., Ghaffari, R., Kim, Y., Lee, S. P., Xu, L., Wu, J., Kim, R., Song, J., Liu Z., Viventi, J., de Graff, B., Elolampi, B., Mansour, M., Slepian, M. J., Hwang, S., Moss, J. D., Won, S., Huang, Y., Litt, B. \& Rogers, J. A. Materials for multifunctional balloon catheters with capabilities in cardiac electrophysiological mapping and ablation therapy. Nat. Mater. 10, 316-323 (2011).

89 Kim, D., Lu, N., Ma, R., Kim, Y., Kim, R., Wang, S., Tao, H., Islam, A., Yu, K. J., Kim, T., Chowdhury, R., Ying, M., Xu, L., Li, M., Chung, H., Keum, H., McCormick, M., Liu, P., Zhang, Y., Omenetto, F. G., Huang, Y., Coleman, T. \& Rogers, J. A. Epidermal electronics. Science 333, 838-843 (2011).

(c)

SOMERIIHIISRESERVE

nported License. To view a copy of this license, visit http:// creativecommons.org/licenses/by-nc-nd/3.0/

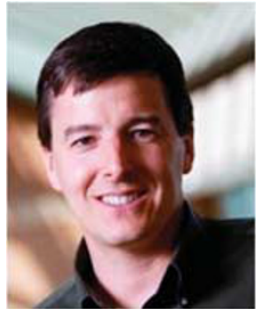

Professor John A Rogers obtained his BA and BS degrees in chemistry and physics from the University of Texas, Austin, in 1989. From MIT, he received SM degrees in physics and chemistry in 1992, and the PhD degree in physical chemistry in 1995. From 1995 to 1997 , Rogers was a Junior Fellow in the Harvard University Society of Fellows. He joined Bell Laboratories as a Member of Technical Staff in the Condensed Matter Physics Research Department in 1997 and served as Director of this department from the end of 2000 to the end of 2002. He is currently the Lee J Flory-Founder Chair in Engineering at University of Illinois at Urbana/Champaign with a primary appointment in the Department of Materials Science and Engineering. Rogers' research includes fundamental and applied aspects of materials and patterning techniques for unusual electronic and photonic devices, with an emphasis on bio-integrated and bio-inspired systems. He has published over 300 papers and is inventor on more than 80 patents, roughly 50 of which are licensed or in active use. Rogers is a member of the National Academy of Engineering and a Fellow of the IEEE, the APS, the MRS and the AAAS. His research has been recognized with many awards, including a MacArthur Fellowship in 2009 and the Lemelson-MIT Prize in 2011.

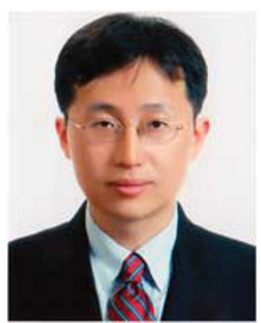

Dr Dae-Hyeong Kim obtained his BS and MS degree in Chemical Engineering from Seoul National University, Korea, in 2000 and 2002 , respectively. He received PhD degree in Materials Science and Engineering from the University of Illinois at Urbana Champaign, in 2009. From 2009 to 2011, he was a post-doctoral research associate at University of Illinois. He is currently an assistant professor in the School of Chemical and Biological Engineering of Seoul National University. He has published more than 32 papers, 22 patents and 1 book chapter. He has been recognized with several awards including George Smith Award (2009) from the IEEE Electron Device Society, MRS Graduate Student Award (Gold Medal, 2009), Green Photonics Award from SPIE (2011) and the TR 35 award from MIT's Technology Review magazine (2011).

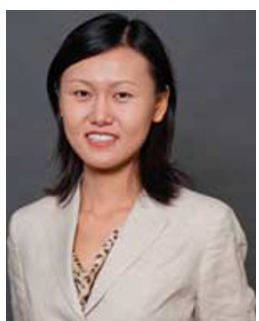

Dr Nanshu Lu received her bachelor's degree in Solid Mechanics from the Department of Engineering Mechanics at Tsinghua University, Beijing in 2005. She obtained her PhD in Solid Mechanics from Harvard University working with Professors Zhigang Suo and Joost Vlassak in 2009. She then received a Beckman Post-doctoral Fellowship and started working on bio-integrated electronics in Professor John Rogers' lab at the University of Illinois at Urbana-Champaign. She joined the Department of Aerospace Engineering and Engineering Mechanics at the University of Texas at Austin as an Assistant Professor in August 2011. Her current research focuses on the mechanics of flexible electronics from material properties to configurational design, and from microfabrication to biointegration, aided by analytical and numerical mechanics modeling. 


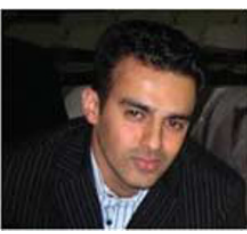

Dr Roozbeh Ghaffari is co-Founder and Senior Program Manager at MC10 Inc, a venture-funded company commercializing biointegrated devices. Dr Ghaffari has also served as a post-doctoral research associate in the Research Laboratory of Electronics at the Massachusetts Institute of Technology. For his PhD research, Dr Ghaffari developed microfluidics and microscale devices to study the micromechanics and electromechanical properties of acellular structures in the inner ear and involved in hearing. His thesis work was awarded the Helen Carr Peake Research Prize (2008) and has been featured on the MIT homepage spotlight and in the Technology Review. Upon completion of his graduate studies, Dr Ghaffari co-authored Diagnostics For All's business plan and identified the organization's leading research program in point-of-care diagnostics. Diagnostics For All won the MIT 100K and Harvard Business School business plan competitions in 2008. Dr Ghaffari holds SB and M Eng degrees in Bio-Electrical Engineering from the Massachusetts Institute of Technology and a PhD degree from the Harvard-MIT Division of Health Sciences and Technology. 